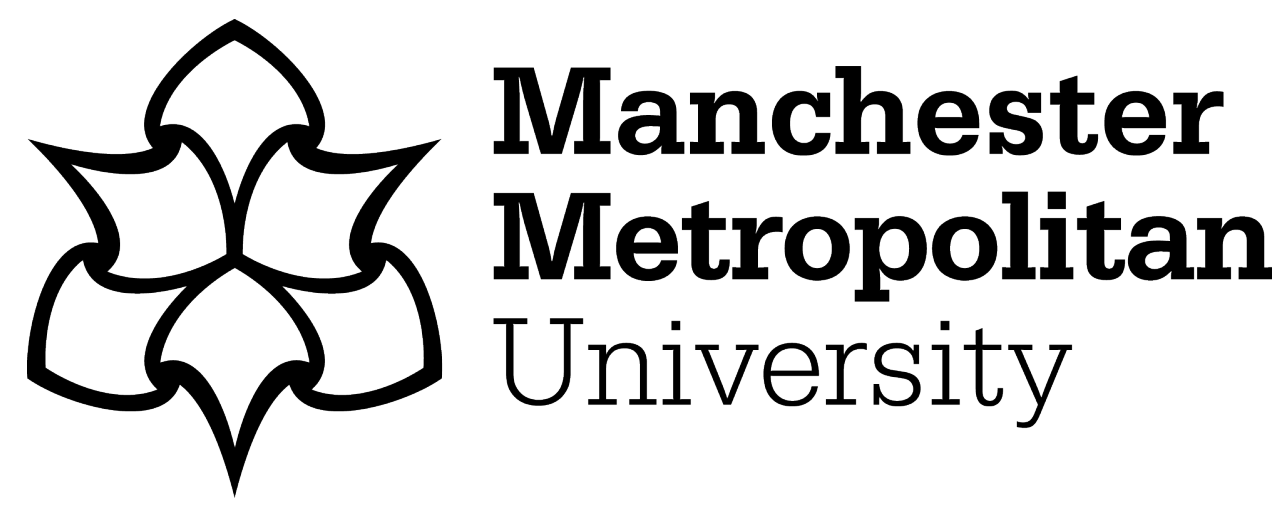

Delhove, JMKM, Karda, R, Hawkins, KE, FitzPatrick, LM, Waddington, SN and McKay, TR (2017) Bioluminescence monitoring of promoter activity in vitro and in vivo. In: Mammalian Synthetic Promoters. Methods in Molecular Biology (1651). Humana Press (Springer Imprint), pp. 49-64. ISBN 9781493972210 (hardcover); 9781493984213 (softcover); 9781493972234 (ebook)

Downloaded from: https://e-space.mmu.ac.uk/619962/

Version: Accepted Version

Publisher: Humana Press (Springer Imprint)

DOI: https://doi.org/10.1007/978-1-4939-7223-4_5

Please cite the published version 


\section{Bioluminescence monitoring of promoter activity in vitro and in vivo}

2

3 Juliette MKM Delhove ${ }^{1,4}$, Rajvinder Karda ${ }^{2}$, Kate E Hawkins ${ }^{1}$, Simon N Waddington ${ }^{2,4}$ \&

4 Tristan R McKay ${ }^{1,3}$

5

$6{ }^{1}$ Cardiovascular \& Cell Sciences Research Institute, St. George's University of London, the Witwatersrand, Johannesburg, South Africa

\section{Summary}

16 The application of luciferase reporter genes to provide quantitative outputs for the 17 activation of promoters is a well-established technique in molecular biology. Luciferase 18 catalyses an enzymatic reaction, that in the presence of the substrate luciferin, produces 19 photons of light relative to its molar concentration. The luciferase transgene can be 20 genetically inserted at the first intron of a target gene to act as a surrogate for the gene's 21 endogenous expression in cells and transgenic mice. Alternatively, promoter sequences can 22 be excised and/or amplified from genomic sources or constructed de novo and cloned 23 upstream of luciferase in an expression cassette transfected into cells. More recently, the 24 development of synthetic promoters where the essential components of an RNA polymerase 25 binding site and transcriptional start site are fused with various upstream regulatory

\section{Key Words}


1 Luciferase, Lentivirus, Promoter, Transcription Factor, Bioluminescence

\section{Introduction}

4 The broad concept of exploiting reporter genes to mark or even quantify gene promoter

5 activity in cells and tissues has traditionally employed bacterially-derived enzymes ( $\beta$ 6 galactosidase and chloramphenicol acetyltransferase -CAT) or an array of fluorescent proteins originating from a myriad of exotic sources. More recently, the ability to quantify promoter activation with greater fidelity and linear range has been made possible by quantifying photonic emission from luciferase activity.

\section{Luciferase reporter genes}

11 The North American firefly luciferase, Photinus pyralis, was the first to be cloned and 12 characterized ${ }^{[1]}$. Firefly luciferase (FLuc) catalyzes an oxidative reaction in the presence of its 13 substrate $\mathrm{D}$-luciferin and adenosine triphosphate (ATP), $\mathrm{Mg}^{2+}$ and $\mathrm{O}_{2}$. The reactive 14 production of the intermediate oxyluciferin releases a photon of light with a quantum yield of 0.88 photons per molecule of luciferin ${ }^{[2]}$. FLuc in particular is a good biomarker as it requires no post-translational modifications, and has a half-life of approximately 3 hours in vitro and in vivo allowing adequate timing for analysis ${ }^{[3]}$. The emission wavelength of FLuc ranges between $530-640 \mathrm{~nm}$, with a peak at $560 \mathrm{~nm}{ }^{[4]}$. Since molecular methods which employ luciferase as a tool occur outside of its natural firefly host, many luciferase constructs have undergone a process of molecular evolution known as codon optimisation. Species-specific codon usage bias results in higher levels of heterologous protein expression due to the enhanced translation processes related to the relative abundance of particular tRNA isoforms ${ }^{[5]}$. For example, a high GC content at the 3rd position of the codon, also known as the silent-site, correlates with an increase in gene expression efficiency within mammalian cells ${ }^{[6]}$. FLuc DNA has been further improved through the removal of sequence repeats, cryptic splice sites, and local hairpin structures to increase mRNA stability ${ }^{[7]}$. Many different luciferase enzymes have been cloned, including those from the sea pansy (Renilla) ${ }^{[8]}$, the railroad-worm (Phrixothrix vivianii) ${ }^{[9]}$, the click beetle (Pyrophorus plagiophthalamus) ${ }^{[9,10]}$, and the jellyfish (Aequorea victoria) ${ }^{[11]}$. Luciferases originating from the sea pansy Renilla reniformis (Rluc), and numerous other ocean-derived organisms oxidize an imidazopyrazinone-based luciferin, coelenterazine, which releases light mostly within the blue-green range at a wavelength of $480 \mathrm{~nm}{ }^{[12]}$ in an ATP-independent reaction.

33 Vargula luciferase is derived from the nocturnal sea ostracod, Vargula hilgendorffii, which 34 naturally inhabits the waters of Japan where it is more commonly known as the "sea firefly". 
1 Historically, vargula has also been named cypridina, owing to the fact that it belongs in the family Cypridinidae ${ }^{[13]}$. In the presence of molecular oxygen and the absence of ATP, vargulin, its substrate, is oxidized and emits light with a peak emission of around $452 \mathrm{~nm}$. This peak emission, however, can shift between 448 and $463 \mathrm{~nm}$ depending on the $\mathrm{pH}$ and salinity of the environment in which the reaction takes place. Interestingly, Thompson et al. showed through sequencing of the vargula gene, that it contains a signal sequence for secretion, and went on to show that the vargula luciferase, secreted from mammalian cells into culture medium, is well suited as a specific and highly sensitive reporter ${ }^{[14]}$. NanoLuc ${ }^{\circledast}$ (NLuc) luciferase is a small luciferase of only $19 \mathrm{kDa}$, isolated from the deep sea shrimp Oplophorus gracilirostris within which it is used as a defense mechanism against predation. The sequence has been isolated and cloned, with the aim of producing a luciferase capable of producing a sustained signal with high sensitivity and low background interference. Following multiple rounds of mutagenesis, this enzyme was engineered to have improved luminescence and stability, and has been codon optimized for mammalian expression. NLuc utilizes the substrate coelenterazine, or ideally its more stable derivative, furimazine, in an ATP-independent reaction. The reaction produces luminescence with an emission peak at $454 \mathrm{~nm}$ and a signal half-life of $>2 \mathrm{~h}$. In contrast to the flash luminescence produced by other luciferases NLuc produces a glow-type luminescence that is not as bright, but can be sustained for hours. Using a secretion signal from the human IL-6 protein, a signal sequence was appended to the $\mathrm{N}$-terminus of the reporter, allowing it to be secreted out of the cell. The secreted NLuc has also been designed to have increased thermal stability, maintaining its enzymatic activity up to $55^{\circ} \mathrm{C}$ and for $>15$ hours at $37^{\circ} \mathrm{C}$ in culture medium [15].

\section{Transcription factor activated luciferase reporters}

26 Luciferase reporter vectors have been widely used in the study of promoter activity after transfection into cell lines. Most often promoters or elements of promoters are cloned upstream of the reporter in order to measure its activity either in steady state or after stimulation by an agonist. More recently the design of synthetic promoters incorporating multiple regulatory sequence motifs has enabled the targeted interrogation of gene enhancer and transcription factor inter-relationships. These experiments are often carried out by plasmid transfection and are thus transient. Synthetic promoters whereby serial minimal transcription factor binding consensus sequences upstream of a minimal polymerase initiating sequence drive reporter activity have been variously described ${ }^{[16]}$. For 
1 example, Pessara et al. first described the application of an NFKB responsive promoter

2 driven reporter to assay TNF $\alpha$ mediated inflammatory responses in a cell model ${ }^{[17]}$. The

3 canonical NFKB (p50/p65) genomic binding has been further defined as 5'-GGGACTTTC-3'

4 and synthetic promoters containing serial repeats of this sequence are dose responsive to

$5 \quad$ NFKB activators. In certain circumstances it may be required to measure transcription factor

6 activity in the long-term or in phenotypically dynamic cells such as stem cells. We recently

7 developed a library of transcription factor activated luciferase reporters cloned into

8 lentiviral vectors ${ }^{[18]}$. We utilised these in the study of cell signalling networks in human

9 dermal fibroblasts as they reprogram to induced pluripotent stem cells ${ }^{[19]}$. This new

10 innovation means that transcription factor activity can be assayed in the long-term in living,

11 changing cells.

12 Building upon these in vitro tools, transgenic mouse strains have been generated in which

13 luciferase expression was controlled by estrogen responsive elements ${ }^{[20]}$ and NF-kB

14 response elements ${ }^{[21]}$, amongst others. However, luciferase activity in the early mouse

15 experiments could only be assayed post-mortem by ex vivo luminometry. The development

16 of highly sensitive charge-coupled device (CCD) cameras has enabled the quantification of

17 luciferase activity in living rodents in a continual and non-invasive manner ${ }^{[22]}$. This has

18 permitted continual measurement of transcription factor activity in live rodents, in

19 numerous models of inflammation ${ }^{[23]}$. However, in germ line transgenic strains it is difficult

20 to distinguish transcription factor activity in specific organs due to the whole-body nature of

21 the transgenesis. Following gene transfer to neonatal mice, using viral vectors, we have

22 demonstrated immune tolerisation ${ }^{[24]}$ and long-term gene expression ${ }^{[25]}$. By exploiting

23 these advantages of neonatal administration, we have been able to apply our library of

24 lentiviral reporter constructs to generate somatic transgenic rodents in which transcriptional

25 activity can be monitored continually and in targeted organs and tissues ${ }^{[18]}$ (See Figure 1). 


\subsection{Construction of lentiviral reporter gene cassette}

1. pLNT-Gateway-MCS was developed as a parental vector by Dr. Steven Howe, UCL and provided as a kind gift.

2. Primers and template to amplify desired reporters. We used both a fluorescent and a luminescent reporter.

\begin{tabular}{|l|l|}
\hline Primer & Sequence (5' --> 3') \\
\hline 3xFLAG (F1) & CTGGGGCCACGAG $\mathbf{~ G A T C C G C C A C C A T G G A C T A C A A A G A C C A T G A C G G T G A T T A T A A A G A T C ~}$ \\
3xFLAG (R1) & TTCTTGGCGTCCTCCATGCTGCCGCCGCCGCTCTTG \\
Fluc (F2) & GCAGCATGGAGGACGCCAAGAACATCAAGAAGGG \\
Fluc (R2) & CTGCGCGGATCTTGCCGCCTTCTTGGC \\
2A-eGFP (F3) & AAGGGCGGCAAGATCCGCGCAGAGGGCCGGGGCTCAT \\
2A-eGFP (R3) & GTCAGCTGGGCAATGCA $\triangle$ TACTAGTTGTGAGTCAAAACTAGAGCCTGGACCA \\
\hline
\end{tabular}

3. High-fidelity polymerase

4. dNTPs

5. PCR cleanup kit

6. $1.5 \%$ agarose gel

7. Gel extraction kit

8. Xho I and Mlu I restriction enzymes

9. Quick Ligase

10. SOC outgrowth media

\subsection{Design and construction of transcription factor activated synthetic promoter}

1. De novo synthesis of minimal promoter and synthetic promoter DNA was performed by Aldevron, Fargo ND, USA

\begin{tabular}{|l|l|}
\hline Primer & Sequence (5' --> 3') \\
\hline $\begin{array}{l}\text { Minimal promoter } \\
\text { (Forward) } \\
\text { Minimal promoter } \\
\text { (Reverse) }\end{array}$ & C $\boldsymbol{\nabla}$ TCGAGGGGCTATAAAAGGGGGTGGGGGCGCGTTCGTCCTCACTCTCTTCCC $\boldsymbol{\nabla}$ TCGAG \\
\hline
\end{tabular}


2. TOP10, DH5 $\alpha$ or similar regular cloning competent cells

3. LB containing kanamycin antibiotic $(50 \mu \mathrm{g} / \mathrm{ml})$

4. Plasmid DNA mini-prep kit

5. T7 primer for sequencing

\subsection{Cloning synthetic promoter into the lentiviral reporter gene cassette}

1. LNT-GW-Luc/eGFP destination vector

2. PENTR-MP vector containing choice of synthetic promoter

3. TE Buffer (pH 8.0)

4. LR clonase II (Invitrogen) (see Note 2)

5. Proteinase $\mathrm{K}$

6. Stbl3 competent cells (see Note $\mathbf{3}$ )

7. SOC outgrowth media

8. $2 x$ ampicillin laden broth and plates $(100 \mu \mathrm{g} / \mathrm{ml})$

\subsection{Generation of high-titer TFAR lentivirus}

1. Highly proliferating, mycoplasma-free HEK293T cells (see Note 4)

2. OptiMem ${ }^{\circledast}$ | reduced-serum medium (Gibco)

3. Phosphate buffered saline (PBS) containing calcium and magnesium (see Note 5)

4. Vasicular Stomatitis Virus glycoprotein (VSV-g) envelope plasmid (pMD2.G)

5. Packaging plasmid containing gag, pol, tat, rev viral genes ( $p C M V \Delta R 8.74)$

6. Transfer plasmid containing the transgene (pLNT-TFAR-Luc/eGFP)

7. Polyethylenimine transfection reagent $(10 \mathrm{mM}): 10 \mathrm{ml}$ branched PEI made up to $41.2 \mathrm{ml}$ with $\mathrm{dH}_{2} \mathrm{O}(\mathrm{pH} 7.0)$ (see Note 6)

8. $0.45 \mu \mathrm{M}$ PVDF sterile filter cups (see Note 7)

9. Complete Media: Dulbecco's Modified Eagles Media, 10\% FBS, 1\% penicillin/streptomycin 
2.5 Lentiviral TFAR transduction of cells

1. Target cell line to be transduced

2. Concentrated lentivirus containing LNT-TFAR-Luc/eGFP

3. Appropriate target cell media

4. Polybrene or alternative cationic polymer for increased transduction

\subsection{In vitro luciferase quantitation to measure TFAR activity.}

1. Opaque, white 96-well plate

\subsection{Neonatal administration of lentiviral TFAR}

\subsection{Continued monitoring of TFAR activity in living mice}

1. D-luciferin (Gold Biotechnology) reconstituted in sterile PBS to a working concentration of $15 \mathrm{mg} / \mathrm{ml}$

2. 27 gauge needle (see Note 11)

3. $1 \mathrm{ml}$ syringe

4. Anesthetic, $100 \%$ Isoflurane inhalation liquid vapor liquid (Abbott)

5. Oxygen $-5.1 \%$, flow rate $1.5 \mathrm{~L} / \mathrm{min}$ 
6. IVIS machine (Perkin-Elmer)

\section{Methods}

\subsection{Construction of lentiviral reporter gene cassette}

1. Primary PCR amplification of individual 3xFLAG-FLuc and 2A-GFP sequences.

2. Secondary, overlap extension PCR performed to anneal and create Fluc-2A-eGFP

\subsection{Design and construction of transcription factor activated synthetic promoter}

1. De novo synthesise minimal promoter (MP) sequence flanked by Xhol sites and clone into unique Xhol site in the multi-cloning site of the pENTR-1A Gateway cloning vector to produce pENTR-MP. We employed the adenoviral E1A minimal promoter.

2. Transform ligated plasmid into chemically competent $\mathrm{DH} 5 \alpha$ E.Coli bacteria by a standard heat shock protocol.

3. Resuspend the transformation solution in $500 \mu \mathrm{ISOC}$ medium and plate out $50 \mu \mathrm{l}$ on LB agar plates containing ampicillin $(30 \mu \mathrm{g} / \mathrm{ml})$ and incubate inverted plates at $37^{\circ} \mathrm{C}$ overnight.

4. Sequence clones to select correctly oriented MP.

5. Derive minimal consensus binding sequence for the candidate transcription factor 
from the literature.

6. Design serial transcription factor binding sequence (TFBS) by interspersing 4-10 binding sequences with 10 random nucleotides.

7. De novo synthesize the resultant sequence with restriction enzyme sites at the $5^{\prime}$ and $3^{\prime}$ - termini. We employ BamHI and EcoRI for pENTR-MP which removes the ccdB and chloramphenicol resistance genes.

8. Directionally clone TFBS into pENTR-MP using TOP10 or DH5 $\alpha$ competent cells to produce pENTR-Prom and grow on kanamycin-laden plates and LB.

9. Confirm clones by sequencing using $T 7$ primer.

\subsection{Cloning synthetic promoter into the lentiviral reporter gene cassette}

1. Set up a recombination reaction by incubating $80 \mathrm{ng}$ of $\mathrm{pENTR}-\mathrm{MP}$ vector containing the desired synthetic promoter with $80 \mathrm{ng}$ of the LNT-GW-Luc vector. (see Note 12)

2. Make up to $4.5 \mu$ l with TE buffer.

3. Mix the Gateway ${ }^{\circledR}$ LR Clonase Enzyme Mix twice for 2 seconds each time and immediately return to $-20^{\circ} \mathrm{C}$ to maintain enzymatic stability.

4. Add $0.5 \mu$ l of the Gateway ${ }^{\circledR}$ LR Clonase Enzyme Mix to the reaction and incubate at $25^{\circ} \mathrm{C}$ for 1 hour. (see Note 13 )

5. Add $1.0 \mu \mathrm{l}$ proteinase $\mathrm{K}$, vortex, incubate at $37^{\circ} \mathrm{C}$ for 15 minutes.

6. Transform $1-1.5 \mu \mathrm{l}$ of this reaction into Stbl3 competent cells (or derivative of).

7. Add $250 \mu \mathrm{l}$ of SOC and allow transformed cells to recover.

8. Plate $50 \mu \mathrm{l}$ of transformed cells on one agar plate $(30 \mu \mathrm{g} / \mathrm{ml}$ ampicillin) and use sterile spreader until all the media has been absorbed into the plate.

9. Spin the remaining competent cells for 30 seconds at $5500 \mathrm{rpm}$.

10. Decant all but $100 \mu$ l of media.

11. Resuspend bacterial pellet and plate onto second ampicillin-containing plate (100 $\mu \mathrm{g} / \mathrm{ml}$ ). (see Note 14)

12. Invert plates and place at $37^{\circ} \mathrm{C}$ overnight.

13. Select and screen colonies for positive clones using the BamHI restriction enzyme and gel electrophoresis. Correct clone contains 5 fragments with the following sizes: 10, 928 bp, 857 bp, 702 bp, 228 bp, 12 bp (not seen on gel). 


\subsection{Generation of high-titer TFAR lentivirus}

1. Seed HEK293T cells at approximately $2 \times 10^{7}$ cells per $T 175 \mathrm{~cm}^{2}$ flask and incubate at $37^{\circ} \mathrm{C}, 5 \% \mathrm{CO}_{2}$ overnight to achieve up to $90 \%$ confluence.

2. Mix $50 \mu \mathrm{g}$ transgene vector, $17.5 \mu \mathrm{g}$ VSV-g envelope vector (pMD2.G), and $32.5 \mu \mathrm{g}$ gag-pol packaging vector ( $p C M V \Delta R 8.74)$ and incubate for 5 minutes at room temperature in $6 \mathrm{ml} \mathrm{OptiMem}^{\circledR} \mathrm{I}$ (Gibco).

3. Add another $6 \mathrm{ml}$ OptiMem ${ }^{\circledR} \mathrm{I}$ containing $1 \mu \mathrm{l}$ polyethylenimine (PEI) $(10 \mathrm{mM})$ and incubate for a further 20 minutes at room temperature.

4. Wash cells with PBS and add $12 \mathrm{mls}$ of PEI/DNA/OptiMem ${ }^{\circledR} \mid$ solution.

5. Incubate at $37^{\circ} \mathrm{C}, 5 \% \mathrm{CO}_{2}$ for 3 hours.

6. Remove PEI/DNA/OptiMem ${ }^{\circledast}$ I solution re-feed cells with DMEM containing $1 \%$ penicillin/ streptomycin and 10\% FCS.

7. After 24 hours, refresh the culture medium.

8. After a further 48 hours collect the virus containing medium and filter through a $0.45 \mu \mathrm{m}$ PVDF filter.

9. Subject virus-containing medium to overnight centrifugation at $4500 \times \mathrm{g}$ at $4^{\circ} \mathrm{C}$.

10. Repeat harvest and overnight centrifugation for 72 hour post-transfection supernatant.

11. As quickly as possible after centrifugation, invert viral supernatant into Virkon ${ }^{\mathrm{TM}}$ to decontaminate the media.

12. Resuspend the viral pellet in $50 \mu$ l OptiMem ${ }^{\circledR} \mathrm{I}$ and gently mix every 20 minutes for 1 hour at $4^{\circ} \mathrm{C}$. (see Note 15)

13. Aliquot viral supernatant into $5-10 \mu \mathrm{l}$ aliquots and transfer to the $-80^{\circ} \mathrm{C}$ freezer as quickly as possible.

14. Lentiviral titer is obtained using a p24 antigen ELISA (Zeptometrix) as per manufacturer's protocol. (see Note 16)

\subsection{Lentiviral TFAR transduction of cells}

1. Passage target cells as per normal maintenance protocol.

2. Transduction is usually carried out once the cells have attached, usually $12-24$ hours post-passaging. (see Note 17) 
3. Remove media, wash cells in PBS. (see Note 18)

4. To a Falcon tube, add the lowest possible volume of target cell growth media required to cover cells.

5. Add TFAR lentivirus at a multiplicity of infection (MOI) of 10 (see Note 19).

6. Incubate cells overnight to allow transduction to take place.

7. Replenish media and continue feeding and passaging processes required for appropriate maintenance of cells.

\subsection{In vitro luciferase quantitation to measure TFAR activity.}

1. Lyse approximately $5 \times 10^{5}$ cells in $300 \mu$ l luciferase lysis buffer and pellet the soluble lysate by high speed centrifugation (13,000 xg for 30 seconds).

2. In triplicate, aliquot $20 \mu \mathrm{l}$ of each cell lysate into a white opaque 96-well plate trying to avoid the pelleted debris.

3. Add $20 \mu$ of luciferase assay buffer, mix and serially inject luciferin substrate into each well to a final concentration of $1.5 \mathrm{mM}$. Luminescence output is measured using an appropriate luminometer with detection parameters in the range of 530$640 \mathrm{~nm}$.

4. Relative photonic light units are normalized relative to total protein as determined by Bradford assay.

5. Statistical analysis using a student's t-test to compare activated vs non-activated samples is used to determine statistical significance between the two groups. 
1. Perform all in vivo injections within twenty-four hours after birth of neonatal mice (see Note 22).

2. Anaesthetize neonatal (PO) CD1 mice on ice.

3. Inject the neonatal mice by the following routes and volumes: intracranially $(5 \mu l)$,

\subsection{Continued monitoring of TFAR activity in living mice}




\section{Notes}

1. $C c d B$ expression results in gyrase-mediated double-stranded DNA breakage, thereby inhibiting bacterial growth. An E.coli mutant strain containing an Arg462 to Cys substitution within the GyrA gene has shown to be resistant to the cytotoxic activity of $c c d B$, and is therefore required for propagation of any clones containing the Gateway cassette.

2. The LR Clonase enzyme is unstable even at $-20^{\circ} \mathrm{C}$ for extended periods. Thus it is recommended that small aliquots of $5 \mu \mathrm{l}$ are made and preferably stored at $-80^{\circ} \mathrm{C}$ to reduce the number of freeze/thaw cycles and retain as much enzyme activity.

3. One Shot ${ }^{\circledast}$ Stbl $^{\text {тM }}$ competent cells have been designed specifically for the propagation of unstable DNA sequence such as those found within the lentiviral backbone which contains direct repeats.

4. Mycoplasma infections have the capacity to reduce lentiviral titers. Therefore, perform a mycoplasma test using a PCR method with the following primers: Forward (5'- gggagcaaacaggattagataccct - 3') and Reverse (5'tgcaccatctgtcactctgttaacctc $-3^{\prime}$ ), or alternatively use a fluorescence based method using a kit such as MycoAlert ${ }^{\mathrm{TM}}$ Mycoplasma Detection kit (Lonza).

5. PBS containing calcium and magnesium improves the adherence of the HEK293T cells during the washing process.

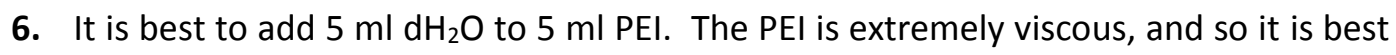
to place the solution in a beaker with a magnetic stirrer. To $\mathrm{pH}$, slowly add $\mathrm{HCL}$ in a drop-wise fashion. This releases fumes from the $\mathrm{HCL}$, and so this step should be carried out in a well-ventilated fume hood. Keep stirring for 2-3 hours in the fume hood. Make up to $20.6 \mathrm{ml}$ final volume with $\mathrm{dH}_{2} \mathrm{O}$, sterilize through a $0.22 \mu \mathrm{M}$ filter and store in $500 \mu \mathrm{l}$ aliquots in the $-80^{\circ} \mathrm{C}$. We have found this concentrated stock to be stable in the fridge for at least 6 months, after which it should be discarded and another aliquot used.

7. Both PES and PVDF filters are suitable but PVDF has been shown to be lower protein binding. If using PES, a $0.22 \mu \mathrm{M}$ filter can be used.

8. Adult female mice will require mating $\approx 20$ days before neonatal mice are required. Ideally this should be timed-mating, where males and females are housed together for one night only (per week).

9. To achieve optimal luciferase expression from the mice, it is best to use white furred 
mice as the black furred mice prevent the bioluminescence from penetrating through.

10. Mice can be injected without anesthesia. However, anesthesia reduces mobility and improves injection accuracy. Mice should only be kept on wet ice until they are immobile. Inhalation or injection anesthetics are avoided as they are associated with a relatively high degree of mortality whereas death from hypothermic anesthesia is very rare.

11. The 33 gauge Hamilton needle should be kept moist at the tip by placing a wet paper towel around the needle. This helps reducing the friction against the new born mouse skin.

12. The site-specific recombination reaction occurs between regions of sequence homology, e.g. the attL sites found flanking the donor sequence within the pENTRProm vector and the attR sites found flanking the Gateway cassette in the acceptor pLNT-FLuc/eGFP vector.

13. It is uncommon, however, if no recombinants have been detected after the first round of recombination, it may be helpful to carry out the recombination reaction using the LR Clonase Enzyme mix for longer than the 1 hour at $25^{\circ} \mathrm{C}$. Instead, the LR reaction can be incubated up to 18 hours (overnight). If the destination vector is $>10 \mathrm{~kb}$ and no recombinants are found following recombination, it is suggested that the destination vector is linearized or a topoisomerase I used to relax the supercoiled DNA prior to incubation with the pENTR and LR Clonase mix.

14. Non-recombinants are dually selected against. Bacteria transformed with the nonrecombined parental vector containing $c c d B$ are killed due to its cytotoxicity in $c c d B-$ sensitive, Stbl3 competent cells, while any non-recombined pENTR vectors are selected against through antibiotic selection as the pENTR-Prom vectors contains $\mathrm{Kan}^{\mathrm{R}}$, while the destination vector contains $A \mathrm{mp}^{\mathrm{R}}$, leading to the growth of bacteria transformed only with recombinants on the ampicillin containing agar plates.

15. Viral pellet may not be visible. The pellet is at the bottom of the Falcon tube and should still be gently resuspended in $50 \mu$ of OptiMem even if it cannot be visually seen.

16. Using this method, make a dilution of $10^{-5}$ or $10^{-6}$ of the concentrated lentiviral supernatant. Dilutions can be made in $\mathrm{dH}_{2} \mathrm{O}$, with $450 \mu \mathrm{l}$ of the final dilution lysed with $50 \mu$ of the supplied lysis buffer.

17. Cells that proliferate quickly and form colonies might be best to transduce directly 
into media following passage. This will allow the cells to be single celled at the time of transduction.

18. Cells may have to be washed more than once if there are a lot of floating cells within the culture. These cells will also be transduced, and therefore will result in lower transduction of the target cells.

19. Cells in suspension or sensitive to cationic polymers can be transduced using centrifugal inoculation (spinoculation) which uses centrifugal force to enhance lentiviral interactions with the cells. This can be performed by spinning the cells in virus-containing media at $800-1200 \times \mathrm{g}$ for 30 minutes at $32^{\circ} \mathrm{C}$ (varies for each cell line) after which the media is aspirated and the cells are resuspended in an appropriate volume of media for their maintenance.

20. If the infection potential of your cell line is not known, use a vector which constitutively expresses the GFP marker gene to ensure that an $\mathrm{MOI}$ of 10 is sufficient to transduce almost all of your cells.

21. VSV-g pseudotyped lentivirus has broad targeting tropism, if however, the cells are difficult to transduce, the addition of a cationic polymer to the media could enhance transduction. Polybrene $(1-8 \mu \mathrm{g} / \mathrm{ml})$ is the most commonly used transduction additive but protamine sulfate $(4-10 \mu \mathrm{g} / \mathrm{ml})$ or poly-L-lysine $(10 \mu \mathrm{g} / \mathrm{ml})$ can also be used.

22. When administering the D-luciferin via intraperitoneal injection, make sure that the bladder or other internal organs are not penetrated by the needle. This can be achieved by "tenting" the skin for injection. Similarly, ensure penetration into the peritoneal cavity by watching for, and avoiding formation of a subcutaneous bleb.

23. Waiting 5 minutes after luciferin administration permits time for entry of the luciferin into the bloodstream. It is worth performing a preliminary experiment to determine kinetics of bioluminescence for different cell and tissue targets. In addition, alternative routes of luciferin administration (e.g. intranasal) may be used [26].

24. Mice may also be anaesthetized using air or air and a nitrous oxide mix. The choice of carrier gas may affect the chosen biosensor as well as firefly luciferase activity (since this is an oxygen-dependent reaction).

25. Image acquisition performed using a $24 \mathrm{~cm}$ field-of-view for greyscale photographic images. Luminescent images are subsequently acquired using a binning factor of 4 , with an aperture of $f / 1.2$ and expressed as photons per second per $\mathrm{cm}^{2}$ per 
steradian. Larger binning values and longer exposures may be necessary if bioluminescence is weak.

26. It is good practice to perform a preliminary experiment to gauge the kinetics of the biosensor response and to identify timeframes of these responses in order to refine subsequent statistical tests. The statistical test and the time points of analysis should be decided before the experiment is performed, not afterwards.

7

\section{Acknowledgements}

9 JMKMD and SNW were funded by the ERC grant Somabio (260862), TRM and SNW were 10 funded by the NC3Rs (NC/L001780/1) and TRM was also funded by EU Horizon2020 grant 11 BATCure (666918). 


\section{References}

2 1. de Wet JR et al (1985) Cloning of firefly luciferase CDNA and the expression of active luciferase in Escherichia coli. Proc Natl Acad Sci U S A 82:7870-3

42 2. Seliger HH, McElroy WD (1964) The Colors of Firefly Bioluminescence: Enzyme Configuration and Species Specificity. Proc Natl Acad Sci U S A 52:75-81

3. Lipshutz GS et al (2001) In utero delivery of adeno-associated viral vectors: intraperitoneal gene transfer produces long-term expression. Mol Ther 3:284-92

4. Rice BW, Cable MD Nelson MB (2001) In vivo imaging of light-emitting probes. J Biomed Opt 6:432-40

5. Shabalina SA, Spiridonov NA, Kashina A (2013) Sounds of silence: synonymous nucleotides as a key to biological regulation and complexity. Nucleic Acids Res 41:2073-94

8. Titushin MS et al (2008) Coelenterazine-binding protein of Renilla muelleri: cDNA

9. Viviani VR, Bechara EJ, Ohmiya Y (1999) Cloning, sequence analysis, and expression

10. Viviani VR et al (1999) Cloning and molecular characterization of the cDNA for the

11. Charbonneau $\mathrm{H}$ et al (1985) Amino acid sequence of the calcium-dependent photoprotein aequorin. Biochemistry 24:6762-71

12. Lorenz WW et al (1991) Isolation and expression of a cDNA encoding Renilla reniformis luciferase. Proc Natl Acad Sci U S A 88:4438-42

13. Morin JG (2011) Based on a review of the data, use of the term 'cypridinid' solves the Cypridina/Vargula dilemma for naming the constituents of the luminescent system of ostracods in the family Cypridinidae. Luminescence 26:1-4 
1 14. Thompson EM, Nagata S, Tsuji FI (1990) Vargula hilgendorfii luciferase: a secreted reporter enzyme for monitoring gene expression in mammalian cells. Gene 96:25762

4 15. Hall MP et al (2012) Engineered luciferase reporter from a deep sea shrimp utilizing a novel imidazopyrazinone substrate. ACS Chem Biol 7:1848-57

16. Criswell $\mathrm{T}$ et al (2003) Transcription factors activated in mammalian cells after clinically relevant doses of ionizing radiation. Oncogene 22:5813-27

17. Pessara U, Koch N (1990) Tumor necrosis factor alpha regulates expression of the major histocompatibility complex class II-associated invariant chain by binding of an NF-kappa B-like factor to a promoter element. Mol Cell Biol 10:4146-54

18. Buckley SM et al (2015) In vivo bioimaging with tissue-specific transcription factor

19. Hawkins KE et al (2016) NRF2 Orchestrates the Metabolic Shift during Induced

20. Ciana P et al (2001) Engineering of a mouse for the in vivo profiling of estrogen

21. Hubbard AK et al (2001) Use of transgenic luciferase reporter mice to determine activation of transcription factors and gene expression by fibrogenic particles. Chest

22. Wu JC et al (2001) Noninvasive optical imaging of firefly luciferase reporter gene

23. Carlsen $\mathrm{H}$ et al (2002) In vivo imaging of NF-kappa B activity. J Immunol 168:1441-6

24. Nivsarkar MS et al (2015) Evidence for contribution of CD4+CD25+ regulatory T cells in maintaining immune tolerance to human factor IX following perinatal adenovirus vector delivery. J Immunol Res 2015:397879

25. Ward NJ et al (2011) Codon optimization of human factor VIII cDNAs leads to highlevel expression. Blood 117:798-807

26. Buckley SM et al (2008) Luciferin detection after intranasal vector delivery is improved by intranasal rather than intraperitoneal luciferin administration. Hum 